\title{
A Lightweight Assessment Method for Medical Device Software Processes
}

\author{
Fergal McCaffery, Paul Clarke, and Marion Lepmets \\ Regulated Software Research Centre, Dundalk Institute of Technology, Dundalk, Ireland \\ \{Fergal.Mccaffery, Paul.Clarke, Marion. Lepmets\}@dkit.ie
}

\begin{abstract}
This paper outlines the MDevSPICE-Adept process assessment method. MDevSPICE-Adept is a lightweight process assessment method that has been created for the MDevSPICE software process assessment model which is currently being developed for the medical device industry. MDevSPICE is a fully validated release of a medical device software process assessment model (formerly known as Medi SPICE), which was developed by the authors. While the MDevSPICE process assessment model is detailed and comprehensive, there is industry demand for a lightweight medical device software process assessment method. To address this requirement the MDevSPICE-Adept method has been developed. Details on how this has taken place and the procedures for implementing an MDevSPICE-Adept process assessment are presented. Information is also provided regarding how an MDevSPICE process assessment was undertaken in an Irish based medical device company. A summary of the issues identified from this process assessment and the actions taken to facilitate process improvement is also presented. Finally, plans for future work are discussed.
\end{abstract}

Keywords: Medical Device Software, Software Process Improvement, Lightweight Process Assessment Method, Medical Device Software Process Assessment, MDevSPICE.

\section{$1 \quad$ Introduction}

Due to the potential threat that medical devices pose to patients, clinicians and third parties their development is highly regulated. In recent years there has been a significant increase in the role and importance that software plays in the healthcare industry [1]. The outcome of this has been the substantial increase in the functionality, complexity and size of software components in medical devices [2]. This development has been recognized by the European Union (EU) in their latest amendment to the Medical Devices Directive (MDD) (2007/47/EC) [3]. As a result, standalone software may now be classified as an active medical device in its own right in the EU. Given the importance and relevance of this measure, the European Commission released a guidance document for the qualification and classification of standalone medical device software MEDDEV 2.1/6 [4] in January 2012. In the United States (US), the Food and Drug Administration (FDA) are responsible for the 
regulation and approval of medical devices and have published software specific guidance documents for medical device software developers, such as the General Principles of Software Validation [5], Off-the-Shelf Software Use in Medical Devices [6] and Guidance on the Content of Premarket Submissions for Software Contained in Medical Devices [7]. To address the increasingly important role that software now plays, the FDA recently published the Medical Device Data Systems Final Rule [8] and Draft Guidance in Relation to Mobile Applications [9].

Given the mission critical nature of medical device software compliance with the relevant regulations, international standards and guidance documents of the region where a medical device is to be marketed is obligatory [10]. In the EU, the receipt of the CE mark is essential and in the US, FDA approval is required. There are approval bodies performing similar roles in other countries including China, Canada, India, Japan, and Australia. A key international standard for achieving regulatory compliance is IEC 62304:2006 [11] and its aligned standards ISO13485:2003 [12], ISO 14971:2007 [13], EN 60601-1:2005 [14], IEC 62366:2007 [15] and IEC 60812:2006 [16]. Information is also provided in relevant technical reports IEC/TR 80002-1:2009 [17] and IEC/TR 61508:2003 [18]. Despite the provision of these international standards, technical reports, regulations and guidance documents, the information they offer is high-level and no specific methods for performing the essential activities required have been provided [19].

It is therefore not surprising, given the importance that achieving regulatory approval plays, that organizations developing medical device software have focused on achieving compliance rather than implementing efficient processes and undertaking process improvement [20]. Previously this was not a critical issue due to the limited proportion of software in medical devices and it was acceptable to take a compliance centric approach. This is no longer the case and there is now a particular requirement for highly effective and efficient software development processes to be in place. These processes need to be defined in a regulatory compliant manner and then adopted to produce the required deliverables in order to achieve approval [19]. To address this requirement, MDevSPICE (formerly known as Medi SPICE [21]), a medical device software process assessment model (PAM) is being developed and validated, which will be made available to the international medical device industry during November 2014. While the MDevSPICE PAM is a comprehensive and detailed process assessment model based on process reference model (PRM) both of which are described in Section 2, there is also industry demand for a lightweight medical device software process assessment method [22]. The MDevSPICE-Adept process assessment method has been developed to help address this requirement and this is discussed in section 3 along with the procedure for its implementation. Section 4 outlines how a MDevSPICE-Adept process assessment was undertaken and provides a summary of the process improvement plan which was collaboratively developed based on the findings report. Section 5 provides a summary and context for future work based on this research. 


\section{$2 \quad$ PRM and PAM of MDevSPICE}

Existing software process models like the Capability Maturity Model Integration (CMMI) [23] and ISO 15504-5:2012 [24] (SPICE) describe generic software development best practices and were not developed to provide coverage of all the necessary areas required to achieve domain specific requirements such as medical device regulatory compliance [25]. To address the requirement for a medical device software development, the Regulated Software Research Centre (RSRC) at Dundalk Institute of Technology (DkIT) undertook extensive research in the area [19]. This resulted in work commencing on the development of MDevSPICE PAM (initially known as Medi SPICE), a medical device specific process assessment model, which is being developed in collaboration with the SPICE community, the international medical device standards community and the international medical device software industry. This process assessment model is in line with Automotive SPICE [26], a domain specific process assessment model for the automotive industry.

The MDevSPICE PAM is based upon the latest version of ISO/IEC 15504-5:2012 and provides coverage of the relevant medical device regulations, standards, technical reports and guidance documents, as illustrated on Fig.1. These include IEC 62304:2006 and its aligned standards (ISO 14971 [13], ISO 13485 [12], IEC TR 80002-1 [17], IEC 62366 [15], IEC 60601-1 [14], IEC 82304 [27]), the FDA guidance documents on premarket submission [7], off-the-shelf software use [6] and software validation [5]. The MDevSPICE PAM is partly founded upon the process reference model for IEC 62304 (IEC TR 80002-3:2014 [28]) as this is the standard for medical device software life cycle processes and is therefore the pivotal standard for medical device software development. It is also worth noting that the development of IEC TR 80002-3 was initiated and lead by the authors (since October 2010) in association with the International medical device standards community combining the requirements from IEC 62304:2006 and ISO/IEC 12207:2008 [29]. Both IEC 62304 and IEC TR 80002-3 describe the process requirements for different software safety classes. This feature has also been carried forward to the MDevSPICE PAM where each process outcome derived from IEC 62304 indicates the safety class for which it is required.

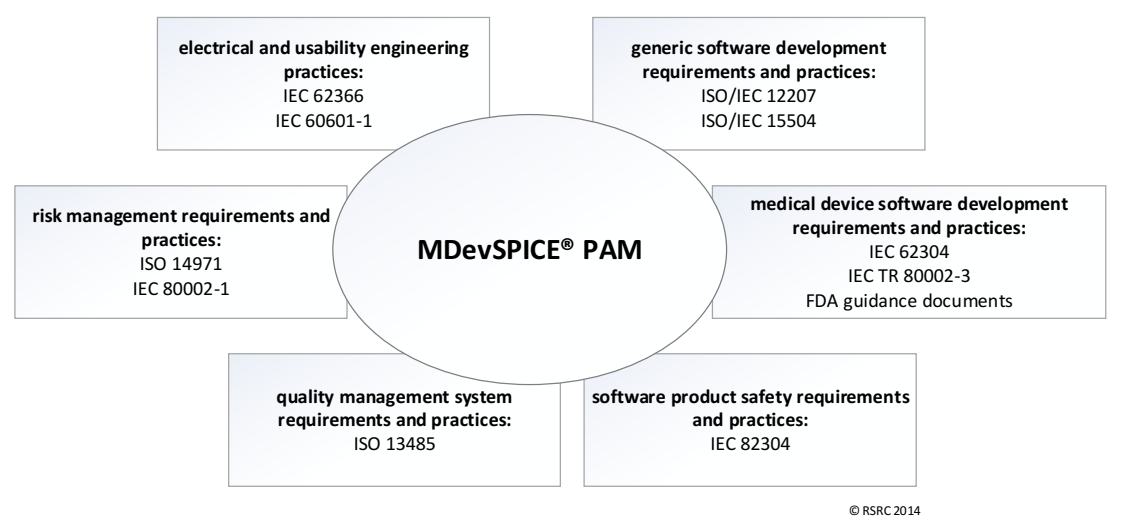

Fig. 1. Collection of requirements and practices of MDevSPICE PAM 
The MDevSPICE PAM contains a Process Reference Model (PRM) which extends the requirements from IEC TR 80002-3 to include requirements from system level and supporting processes described in ISO/IEC 12207:2008. The MDevSPICE PRM consists of 24 processes which are fundamental to the development and maintenance of regulatory compliant medical device software. Each process has a clearly defined purpose and process outcomes that must be accomplished to achieve that purpose. One way to achieve these process outcomes is through implementing the base practices described in the MDevSPICE PAM. The MDevSPICE PAM extends the PRM with additional process elements of base practices and work products and the measurement framework allowing process capability ratings. Similarily to ISO/IEC 15504-5, the MDevSPICE PAM also has two-dimensional view of process capability. In one dimension, it describes a set of base practices that allow the achievement of the process outcomes and purpose defined in the PRM; this is termed the process dimension. In the other dimension, the PAM describes capabilities that relate to the process capability levels and process attributes, this is termed the capability dimension.

The MDevSPICE PAM extends the PRM with a set of work products for every process that are the inputs and outputs of the processes. In ISO/IEC 15504-5, work products are the informal evidence collected to support the process capability rating. In MDevSPICE PAM, these are both informal as well as normative, i.e. mandatory work products as required in the regulatory standards. The existence of these mandatory work products together with their required content are addressed as process outcomes during the process assessment.

The MDevSPICE PAM also includes the measurement framework, which is based on ISO/IEC 15504-2:2003 [30]. Similarly to ISO/IEC 15504-2, the MDevSPICE PAM also has six process Capability Levels, with one or more process attributes per Capability Level from Level 1 onwards.

The objective of undertaking an MDevSPICE process assessment is to determine the state of a medical device organisation's software processes and practices in relation to the regulatory requirements of the industry and to identify areas for process improvement [31]. It can also be used as part of the supplier selection process when an organisation wishes to outsource or offshore part or all of their medical device software development to a third party or remote division [32]. An MDevSPICE process assessment can also be conducted for pre-qualification purposes as it provides a preliminary readiness overview of regulatory compliance.

The MDevSPICE PRM and PAM were released in stages and each stage was extensively reviewed by interested parties from the SPICE community, representatives from international standards bodies and medical device industry experts. This collaborative approach is seen as a key element in the development of the MDevSPICE PAM to ensure coverage of both the generic software best practices and medical device software regulatory requirements [31]. The MDevSPICE PAM is a comprehensive and detailed process assessment model and its overall objective is to provide both process capability and conformity assessment ratings to support first, second or third party assessments. It is envisaged that results from these assessments may be recognized by the relevant regulatory bodies. 


\section{Requirements for MDevSPICE-Adept Process Assessment Method}

As outlined in section two, there is a specific requirement for a detailed and comprehensive process assessment model, which is specific to the medical device domain and that MDevSPICE PAM is addressing. as with other process models i.e. CMMI and IEC 15504-5:2012. A full MDevSPICE process assessment will require considerable planning and resources to successfully undertake. While MDevSPICE process assessment is being developed with the objective of being as efficient as possible, the necessity for rigour dictates the level of planning, resources and analysis required for its successful implementation. While the need for and importance of MDevSPICE process assessment is understood [21], it was also appreciated by the RSRC that there is a specific requirement for a lightweight assessment method in the medical device software industry [33]. In particular, there was industry led demand for a lightweight assessment method based on the MDevSPICE PAM. This was communicated directly to the RSRC by numerous medical device organisations. To address this specific requirement, the MDevSPICE-Adept method was developed. This also provided an opportunity to leverage the extensive research [19] and level of detail, which developing the MDevSPICE PAM required.

A process assessment method provides a process description for conducting process assessments. The rigour of the method depends on the purpose of the process assessment. For Class 3 third-party assessment, the rigour is highest i.e. the amount of evidence collected and analysed is greatest, and assessor competence and experience highest. The more rigorous the chosen process assessment method, the more detailed are the process assessment results. On the other hand, the more rigorous the method, the more resource-demanding and time-consuming is the process assessment.

To be effective, MDevSPICE-Adept required the employment of a lightweight approach for undertaking software process assessment and improvement. This included the use of a limited number of personnel to carry out and participate in the assessment while also maximising the benefit of the time and effort of those involved. It was envisaged that MDevSPICE process assessment would eventually encompass all the MDevSPICE PAM processes. It was therefore recognized that an assessment could take place over a day or a number of days depending on how many processes were being assessed. It was also important that organizations could select the specific processes which were of most benefit for achieving their business goals. The focus of the method had to be on the evaluation of the essential base practices, key work products and the achievement of the process outcomes which were necessary for the attainment of the specific process purpose being assessed. MDevSPICE-Adept therefore needed to be process dimension centric in its focus. Finally, the objective of undertaking an MDevSPICE-Adept process assessment was not to receive formal certification, but rather to identify an organization's strengths and weaknesses and to facilitate process improvement. Having defined the criteria which had to be met, the next step was to undertake the development of MDevSPICE-Adept method. 


\subsection{Developing the MDevSPICE-Adept Method}

The RSRC, having previously successfully developed and implemented three lightweight software process assessment methods Adept [33], Med-Adept [34] and Med-Trace [35], the objective was to leverage that experience and utilise it for the development of MDevSPICE-Adept. It was in this context that work commenced on the development of MDevSPICE-Adept. It was recognized that this process assessment method needed to cover more processes and provide more detailed analysis than those methods which had been previously developed. While this was the case, MDevSPICE-Adept was still required to be lightweight to satisfy the industry demand. The MDevSPICE-Adept method was developed through process assessment engagements in medical device industry.

The first task was to identify the initial set of processes that would be included in the MDevSPICE process assessment. The goal was to select a limited number of processes that would be most beneficial and relevant to industry with the onsite process assessment no longer than 2 days. To achieve this, industry experts were consulted on the 24 processes of the PAM from which they selected the preliminary 11 processes (most of which are from IEC 62304):

- System requirements analysis

- Software development planning

- Software requirements analysis

- Software architectural design

- Software detailed design

- Software unit implementation and verification

- Software integration and integration testing

- Software system testing

- Software risk management

- Software configuration management

- Software problem resolution

While these were the initial processes selected for inclusion in MDevSPICEAdept, it is also possible to extend this list of processes to provide coverage of all the MDevSPICE processes. This can only be done with an extended onsite process assessment demanding more time and resources from the company that is being assessed.

The MDevSPICE PAM had been developed for each of the initial processes which were based on best practice as outlined by the latest version of ISO/IEC 15504-5 and the specific requirements of the medical device regulations, standards, technical reports and guidance documents. As a result, each process had a defined purpose and process outcomes, base practices and work products were also included for the achievement of these process outcomes and process purpose. In addition, each outcome and base practice was cross referenced to the standard they were derived from. To facilitate the process assessment, each of the initial processes were evaluated and specific questions identified based on the MDevSPICE PAM. This work was undertaken by six members of the RSRC team with extensive experience in SPI and 
knowledge of medical device software development. Having defined the assessment instrument, the next step was to develop the specific process for undertaking an MDevSPICE assessment.

The objective of MDevSPICE-Adept is to assist an organisation to gain an understanding of the state of their current software development processes when measured against the selected MDevSPICE PAM processes. The MDevSPICE PAM is essentially a "one stop shop" for all associated medical device software related requirements. The MDevSPICE PAM contains the requirements of all the software related medical device standards and the additional software engineering best practices from ISO/IEC 15504-5. Even though the MDevSPICE process assessment does not provide any official certification against any of the medical device standards, the output of the assessment will provide both the MDevSPICE process capability rating as well as the gaps that exist against the requirements of the medical device software standards included in the MDevSPICE PAM. This type of combinatory process assessment result allows targeted activities for both process improvement and an increased regulatory compliance to be undertaken by the assessed organization.

\subsection{The Procedure for Undertaking a Lightweight MDevSPICE Process Assessment Following the MDevSPICE-Adept Method}

Based on the RSRC's previous experience of developing and undertaking lightweight software process assessments [33], the seven stage procedure for undertaking an MDevSPICE Assessment following the MDevSPICE-Adept method was defined. It was decided the assessment team should normally consist of two assessors who share responsibility for conducting the assessment.

The seven stages of the procedure are as follows: As a precursor to undertaking an assessment a preliminary meeting between the lead assessor and the company takes place. This is the first stage in the procedure and during this meeting the lead assessors discusses the main drivers for the company wishing to undertake an assessment. In this context the expectations regarding what can be realistically achieved are discussed and the procedure for undertaking the assessment is outlined. If there is agreement a schedule is drawn up.

At the second stage the lead assessor has a meeting with the staff and management from the company who will be participating in the assessment where an overview of the MDevSPICE-Adept assessment method is presented and details of what their participation will involve is outlined.

On the agreed date the onsite assessment commences which is the third stage in the procedure. For each process the lead assessor conducts interviews based on the scripted MDevSPICE-Adept questions with the relevant personnel and evaluates the responses. The second assessor who also participates in the interviews prepares interview notes and may ask additional questions when clarification is required. In addition work products may also be requested and reviewed as part of this stage. A maximum of five processes are assessed in a single day with the interviews for each process taking approximately one hour. 
At the fourth stage the findings report is prepared off site based on the data gathered at stage three. Each process is reviewed in turn and relevant strengths and issues (weaknesses) are identified based on the evaluation and interview notes. Suggested actions to address these issues and to facilitate process improvement are outlined and discussed. The possibility for the use of appropriate agile and lean practices is also considered. These are then documented and included in the findings report. This is a joint effort between the assessors and may include other SPI and/or lean and agile experts if required.

The findings report is then presented to the management and staff who took part in the assessment which is the fifth stage in the procedure.

Having provided adequate time for the findings report to be read and considered by the organization, at the sixth stage the contents of the report is discussed in detail with the relevant management and staff. At this point specific objectives for process improvement are collaboratively defined based on the findings report which results in the development of a process improvement plan. Given the lightweight nature of MDevSPICE-Adept improvements that offer the greatest benefits in terms of compliance, quality and the achievement of business goals are selected for inclusion in this plan.

At the seventh stage in the procedure the organization having implemented the process improvement plan have the opportunity of having the processes reassessed. Based on this, a final detailed report is prepared which highlights what has been achieved and an updated improvement plan is also provided.

\section{Conducting the Lightweight MDevSPICE Process Assessment Following the MDevSPICE-Adept Method}

Having developed the MDevSPICE-Adept Assessment method and the procedure for its implementation the first assessment took place in an Irish based medical device company Irish Medical (a pseudonym). The company develops both automotive and medical device software. Each of their products contains both hardware and software and the role that software plays has considerably increase over the last number of years. They produce software for medical devices that will be marketed in the EU and the US so their products must conform to the MDD to receive the CE mark and the FDA regulations.

Having agreed that an assessment would take place (Stage 1), it was decided by the company that 10 out of the 11 processes would be assessed over a two day period (Stage 2). Software problem resolution process was omitted as an explicit process for managing problems did not exist in the Irish Medical at the time of the process assessment. The process assessment, i.e. Stage 3, was undertaken by two assessors from the RSRC. Based on the results of the process assessment, a findings report was prepared and presented in Stage 4. The focus of the report was that for each process the strengths and issues were highlighted, in addition suggested actions to facilitate process improvement were provided. Based on the findings report, the process improvement objectives and process improvement plan were collaboratively defined 
and developed with the company (Stage 5-6). A summary of the issues identified for each process and the actions taken to address these issues and facilitate improvement were outlined in the process improvement plan of Stage 7.

Irish Medical decided that the 10 critical processes to be assessed are the following:

- System requirements analysis

- Software development planning

- Software requirements analysis

- Software architectural design

- Software detailed design

- Software unit implementation and verification

- Software integration and integration testing

- Software system testing

- Software risk management

- Software configuration management

All of the 10 processes are described in IEC 62304 and IEC TR 80002-3 with the exception of System requirements analysis. This is an important process for Irish Medical as it develops software for embedded medical device systems and requires efficient traceability of requirements from system level to software level.

In conducting the process assessment in Irish Medical, it was possible to not just highlight strengths and weaknesses in the process implementation, but it was also possible to tailor the scope of the process assessment to suit the needs of the organisation. Fig 2 below demonstrates the coverage of the process assessment from the underlying standards perspective. In practice, additional standards can be added to full MDevSPICE assessments but for the purpose of this MDevSPICE-Adept process assessment, this coverage was deemed sufficient.

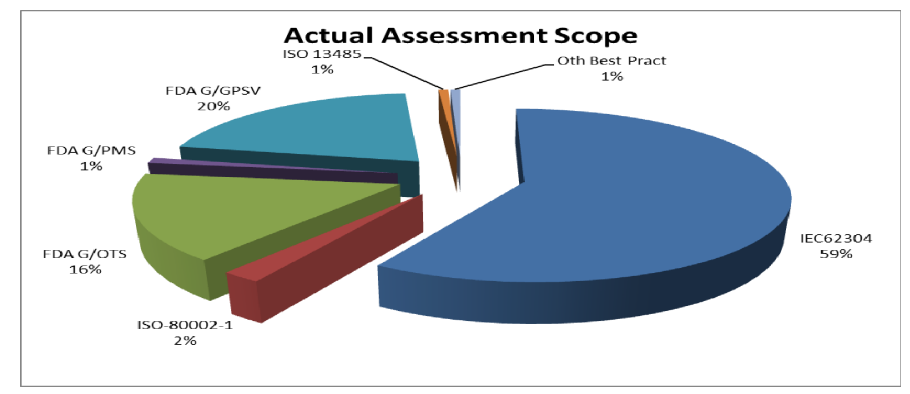

Fig. 1. Scope of Irish Medical process assessment from the underlying standards perspective

One of the primary observations from this process assessment was the extent to which the basic requirements of IEC 62304 were in fact supplemented with best practice know-how from a range of other standards, thus resulting in a more thorough evaluation of the software development process capability. It is furthermore the case that for each of the underlying sources, it is possible to also produce findings in 
relation to coverage of that particular standard - and not just for individual processes but for groups of processes and the process set as a whole. For example, Figure 3 demonstrates how an overall coverage of software development process requirements for safety class C software development based on IEC 62304 might appear.

At an individual process level, the participating company was provided with detailed information on the process capability rating and areas of greatest weakness. One example of such related to software configuration management, an area that the organisation is now actively addressing.

The findings report was positively received by Irish Medical as was the whole process assessment. The collaborative nature of the development of the process improvement plan provides motivation for it successful implementation. The plan is currently being implemented and when this is complete the opportunity to have the processes reassessed is available - and the company in question is positively predisposed to introducing a regular process assessment in order to better understand and improve their software processes. The process assessment also identified areas of strength in the organisation. For example, the company had very good risk management and traceability procedures in place. It is important to state that the MDevSPICE-Adept process assessment method highlights the strengths as well as the weaknesses in an organization.

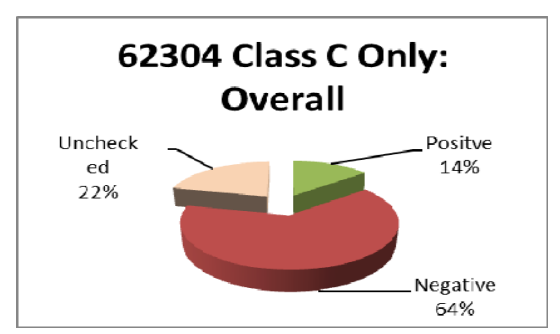

Fig. 2. Sample IEC 62304 safety class C coverage

\section{Conclusion}

The MDevSPICE framework consists of both the MDevSPICE PAM and the MDevSPICE-Adept process assessment method. The MDevSPICE PAM is a comprehensive and detailed domain specific process assessment model for medical device software development. It provides the basis for in-depth analysis and assessment of each process including the measurement framework for process capability determination. As a result, the findings from a Class 3 MDevSPICE process assessment can be comprehensive and detailed. On the other hand, MDevSPICE-Adept as a Class 1 lightweight assessment method has a different purpose. Its focus is high level and its role is to provide a snap shot of medical device software development processes, and to assist with regulatory compliance and process improvement in this context. 
MDevSPICE-Adept is the largest and most detailed of the lightweight assessment methods developed by the RSRC. It is the result of industry demand and was developed to meet the requirement for an extensive yet lightweight medical device software assessment method. A pilot MDevSPICE-Adept assessment has recently been successfully implemented in Ireland. Feedback from the assessment was very positive. In line with our strategy for MDevSPICE-Adept method, we will develop various sets of initial processes based on the different demands of medical device software development organizations based on compliance requirements with specific standards. Given the level of demand it is also our objective to carry out additional MDevSPICE-Adept assessments both in Ireland and in collaboration with our international colleagues. We also plan to release Class 1 and Class 2 MDevSPICE process assessment methods in November 2014.

Acknowledgments. This research is supported by Enterprise Ireland and the European Regional Development Fund (ERDF) under the National Strategic Reference Framework (NSRF) 2007-2013, grant number CF/2012/2631, and in part by the Science Foundation Ireland (SFI) Stokes Lectureship Programme, grant number 07/SK/I1299, and the SFI Principal Investigator Programme, grant number 08/IN.1/I2030, and in part by Lero.

\section{References}

1. Abraham, C., Nishiharas, E., Akiyama, M.: Transforming healthcare with information technology in Japan: A review of policy, people, and progress. International Journal of Medical Informatics 80(3), 157-170 (2011)

2. Lee, I., Pappas, G., Cleaveland, R., Hatcliff, J., Krogh, B., Lee, P., Rubin, H., Sha, L.: High-Confidence Medical Device Software And Systems. Computer 39(4), 33-38 (2006)

3. European Council, Council Directive 2007/47/EC (Amendment). In: Official Journal of The European Union: Luxembourg (2007)

4. European Commission, MEDICAL DEVICES: Guidance document- Qualification and Classification of stand alone software (MEDDEV 2.1/6). Brussels, Belgium (2012)

5. US FDA Center for Devices and Radiological Health, General Principles of Software Validation; Final Guidance for Industry and FDA Staff. CDRH: Rockville (2002)

6. US FDA Center for Devices and Radiological Health, Off-The-Shelf Software Use in Medical Devices; Guidance for Industry, medical device Reviewers and Compliance. CDRH: Rockville (1999)

7. US FDA Center for Devices and Radiological Health, Guidance for the Content of Premarket Submissions for Software Contained in Medical Devices. CDRH: Rockville (2005)

8. US FDA, 21 CFR Part 880 Medical Devices; Medical Device Data Systems Final Rule. Federal Register, 2011. vol. 76(31), pp. 8637 - 8649 (2011)

9. US FDA, Draft Guidance for Industry and Food and Drug Administration Staff Mobile Medical Applications (2011)

10. Vogel, D.A.: Medical Device Software Verification, Validation and Compliance, p. 432. Artech House, Norwood (2010) 
11. IEC 62304:2006, Medical device software-Software life cycle processes. IEC: Geneva, Switzerland (2006)

12. ISO 13485:2003, Medical devices — Quality management systems - Requirements for regulatory purposes. ISO: Geneva, Switzerland (2003)

13. ISO 14971:2007, Medical Devices - Application of risk management to medical devices. ISO: Geneva, Switzerland (2007)

14. BS EN 60601-1:2005Medical electrical equipment - Part 1: General requirements for basic safety and essential performance. IEC: Geneva, Switzerland (2005)

15. IEC 62366:2007, Medical devices - Application of usability engineering to medical devices. IEC: Geneva, Switzerland (2007)

16. IEC 60812:2006, Analysis technique for system reliability - Procedure for failure modes and effects analysis (FMEA). IEC: Geneva, Switzerland (2006)

17. IEC/TR 80002-1:2009, Medical device software Part 1: Guidance on the application of ISO 14971 to medical device software. BSI: London (2009)

18. IEC/TR 61508:2005, Functional safety of electrical/electronic/ programmable electronic safety related systems. BSI: London (2005)

19. Mc Caffery, F., Burton, J., Casey, V., Dorling, A.: Software Process Improvement in the Medical Device Industry. In: Laplante, P. (ed.) Encyclopedia of Software Engineering, pp. 528-540. CRC Press Francis Taylor Group, New York (2010)

20. Denger, C., Feldmann, R., Host, M., Lindholm, C., Shull, F.: A Snapshot of the State of Practice in Software Development for Medical Devices. In: First International Symposium on Empirical Software Engineering and Measurement, Madrid, Spain (2007)

21. Mc Caffery, F., Dorling, A.: Medi SPICE: An Overview. In: International Conference on Software Process Improvement and Capability Determinations (SPICE), Finland (2009)

22. Casey, V., Mc Caffery, F.: Med-Trace: Traceability Assessment Method for Medical Device Software Development. In: European Systems and Software Process Improvement and Innovation Conference. Roskilde University, Denmark (2011)

23. CMMI Product Team, Capability Maturity Model Integration for Development Version1.3. Software Engineering Institute, Pittsburg PA (2010)

24. ISO/IEC 15504-5:2012, Information technology - Process Assessment - Part 5: An Exemplar Software Life Cycle Process Assessment Model. ISO (2012)

25. Mc Caffery, F., Dorling, A.: Medi SPICE Development. Software Process Maintenance and Evolution: Improvement 22(4), 255-268 (2010)

26. Automotive SIG, Automotive SPICE Process Assessment V 2.2 (August 21, 2005)

27. IEC/CD 82304:2014, Health Software - Part 1: General Requirements for Product Safety. ISO: Geneva, Switzerland (2014)

28. IEC/TR 80002-3:2014, Medical Device Software - Part 3: Process reference model for medical device software life cycle processes (IEC 62304). ISO: Geneva, Switzerland (2014)

29. ISO/IEC 12207:2008, Systems and software engineering - Software life cycle processes. ISO: Geneva, Switzerland (2008)

30. ISO/IEC 15504-2:2003, Software engineering - Process assessment - Part 2: Performing an assessment, ISO: Geneva, Switzerland (2003)

31. Mc Caffery, F., Dorling, A., Casey, V.: Medi SPICE: An Update. In: International Conference on Software Process Improvement and Capability Determinations (SPICE), Pisa, Italy (2010)

32. Casey, V.: Virtual Software Team Project Management. Journal of the Brazilian Computer Society 16(2), 83-96 (2010) 
33. Mc Caffery, F., Richardson, I., Coleman, G.: Adept - A Software Process Appraisal Method for Small to Medium-sized Irish Software Development Organisations. In: European Systems \& Software Process Improvement and Innovation (EuroSPI 2006), Joensuu, Finland (2006)

34. Mc Caffery, F., Casey, V.: Med-Adept: A Lightweight Assessment Method for the Irish Medical Device Software Industry. In: European Systems \& Software Process Improvement and Innovation Conference (EuroSPI), Grenoble, France (2010)

35. Casey, V., Mc Caffery, F.: A lightweight traceability assessment method for medical device software. Journal of Software Maintenance and Evolution Research and Practice (2011) 